\title{
A SUFFICIENT CONDITION FOR HYPERBOLICITY OF PARTIAL DIFFERENTIAL OPERATORS WITH CONSTANT COEFFICIENT PRINCIPAL PART
}

BY

\author{
JOSEPH L. DUNN $\left.{ }^{1}\right)$
}

\begin{abstract}
Let $\boldsymbol{P}$ be a differential operator with principal part $\boldsymbol{P}_{\boldsymbol{m}}$, and suppose that $\boldsymbol{P}_{\boldsymbol{m}}$ has constant coefficients and is hyperbolic. It is shown that the condition for hyperbolicity of $P$ when $P$ has constant coefficients, namely, that $P$ is weaker than $P_{m}$ is also a sufficient condition for hyperbolicity in the case where $P$ does not have constant coefficients. Some generalizations are also made to the case where $P$ is a square matrix of differential operators.
\end{abstract}

1. Introduction. Consider a partial differential operator $P(D)=$ $\Sigma_{|\alpha| \leqslant m} a_{\alpha}(t, x) D^{\alpha}$ of order $m$ in $n+1$ independent variables where $\alpha$ is a multi-index $\left(\alpha_{0}, \alpha_{1}, \cdots, \alpha_{n}\right)$ and

$$
D^{\alpha}=\left(\frac{1}{i} \frac{\partial}{\partial t}\right)^{\alpha_{0}} \cdots\left(\frac{1}{i} \frac{\partial}{\partial x_{n}}\right)^{\alpha_{n}} .
$$

There is as yet no general theory which allows one to make the assertion that such an operator is hyperbolic iff the $a_{\alpha}$ satisfy certain conditions. The traditional definition says that an operator is hyperbolic iff its principal part $P_{m}(\xi)=$ $\Sigma_{|\alpha|=m^{a} \xi^{\alpha}}$ is such that $P_{m}(\xi+\tau N)=0$ for $\xi$ real and $N=(1,0)$ implies that $\tau$ is real. However, at best this should be regarded as a necessary condition. This can be best illustrated by considering the case of constant coefficients where there has been developed a good theory of hyperbolicity.

In this case one says that a differential operator $P$ is hyperbolic with respect to a covector $N$ if there exists a fundamental solution $E$, i.e., a solution of the equation $P(D) E=\delta$, such that supp $E$ is contained in a proper cone, i.e., a closed cone contained in the set $H \cup\{0\}$ where $H=\{x:\langle x, N\rangle\rangle 0\}$.

Received by the editors February 2, 1974.

AMS (MOS) subject classifications (1970). Primary 35L25, 35L30, 35L55; Secondary 35B45.

Key words and phrases. Hyperbolic partial differential equation, energy integral, principal part with constant coefficients.

(1) Supported by a National Science Foundation Graduate Fellowship. 
Gårding [2] was able to obtain a simple algebraic characterization of hyperbolicity, namely $P$ is hyperbolic with respect to $N$ iff $P_{m}(N) \neq 0$ and $P(\xi+\tau N)$ $\neq 0$ for $\xi$ real and $|\operatorname{Im} \tau|>c, c$ a constant. To be more precise about the sufficiency of this condition one needs the following:

Definition 1.1. If $P$ is hyperbolic w.r.t. $N$, and $P_{m}$ is the principal part of $P$, we denote by $\Gamma(P, N)$ the set of all real $\theta$ such that the polynomial $P_{m}(\theta+\tau N)$ has only negative roots $\tau$.

We can now state the following.

THEOREM 1.2 (HÖRMANDER [4, P. 137]). Let $P(D)$ satisfy Gårding's condition for hyperbolicity with respect to $N$. Then there exists one and only one fundamental solution $E$ of the operator $P(D)$ with support in the half space $H=\{x:\langle x, N\rangle \geqslant 0\}$. For this fundamental solution we have supp $E$ is contained in the convex cone

$$
\Gamma^{*}(P, N)=\{x:\langle x, \theta\rangle \geqslant 0, \theta \in \Gamma(P, N)\}
$$

but in no smaller convex cone with vertex at 0.

One can show that if $P$ is hyperbolic then so is its principal part $P_{m}$ and that in order for $P_{m}$ to be hyperbolic the equation $P_{m}(\xi+\tau N)=0$ must have only real roots $\tau$ for $\xi$ real. However, one does not have in general that $P$ is hyperbolic if $P_{m}$ is. In order to determine the precise relationship between the hyperbolicity of $P$ and that of $P_{m}$ one needs the following.

Definition 1.3. A polynomial $P$ is said to be stronger than a polynomial $Q$ iff

$$
\left(\sum_{\alpha}\left|D_{\xi}^{\alpha} Q(\xi)\right|^{2}\right)^{1 / 2} \leqslant C\left(\sum_{\alpha}\left|D_{\xi}^{\alpha} P(\xi)\right|^{2}\right)^{1 / 2}
$$

One can now state the following.

THEOREM 1.4 (Hörmander [4] AND SVENSSON [8]). If the principal part $P_{m}$ of $\boldsymbol{P}$ is hyperbolic w.r.t. $N$, then $\boldsymbol{P}$ is hyperbolic w.r.t. $N$ iff $\boldsymbol{P}$ is weaker than $P_{m}$. In this case $P$ and $P_{m}$ are equally strong.

So much for the case of constant coefficients. The reason for its complete success is that when one Fourier transforms a differential operator with constant coefficients one obtains a polynomial. Obviously one is not so lucky in the case of variable coefficients. Certain other cases, namely the cases where $P$ has simple characteristics or is a symmetric first order operator, can be handled by the method of energy integrals. Peyser [6] considers the extension of energy integral methods to the case where $P$ has constant coefficients. He found that the method worked when the polynomial satisfied a condition which he called proper hyperbolicity. 
Svensson [8] was then able to show that an operator is properly hyperbolic iff it is hyperbolic.

In order to explain these conditions we assume that there is an inner product on the space of independent variables and that $P(\tau, \xi)=P(\tau N+\xi)$ for $\xi$ real and in $N^{\perp}$. Then the hyperbolicity of $P$ w.r.t. $N$ is equivalent to $P_{m}(1,0) \neq 0$ and $P(\tau, \xi) \neq 0$ for $\xi$ real and $|\operatorname{Im} \tau|>c$. In particular the principal part of $P, P_{m}$, is hyperbolic w.r.t. $N$ iff $P_{m}(1,0) \neq 0$ and $P_{m}(\tau, \xi)=0$, $\xi$ real implies $\tau$ is real.

Assume now that $\boldsymbol{P}_{\boldsymbol{m}}$ is hyperbolic. Then we have

$$
P_{m}(\tau, \xi)=\prod_{k=1}^{m}\left(\tau-\lambda_{k}^{m}(\xi)\right)
$$

where $\lambda_{k}^{m}(\xi)$ is real for $\xi$ real and we have assumed without loss of generality that the coefficient of $\tau^{m}$ is 1 .

Now define

$$
P_{m}^{(i)}(\tau, \xi)=\frac{\partial^{i}}{\partial \tau^{i}} P_{m}(\tau, \xi)=m(m-1) \cdots(m-i+1) \prod_{k=1}^{m-i}\left(\tau-\lambda_{k}^{m-i}(\xi)\right)
$$

where again one has $\lambda_{k}^{m-i}(\xi)$ real for $\xi$ real.

Define also

$$
\left(P_{m}^{(i)}\right)_{j}=\frac{P_{m}^{(i)}(\tau, \xi)}{\tau-\chi_{j}^{m-i}}=m(m-1) \cdots(m-i+1) \prod_{k=1 ; k \neq j}^{m-i}\left(\tau-\lambda_{k}^{m-i}(\xi)\right) .
$$

One now has

THEOREM 1.5 (Svensson [8]). Suppose $P(\tau, \xi)=\Sigma_{i=0}^{m} P_{i}(\tau, \xi)$ where $P_{i}(\tau, \xi)$ is a polynomial homogeneous of degree $i$, and suppose that $P_{m}(\tau, \xi)$ is hyperbolic and that the coefficient of $\tau^{m}$ is 1 , then $P$ is hyperbolic iff

$$
P_{m-i}(\tau, \xi)=\sum_{j=1}^{m-i+1} \gamma_{i}^{j}(\xi)\left(P_{m}^{(i-1)}\right)_{j}(\tau, \xi), \quad i \geqslant 1
$$

where the $\gamma_{i}^{j}(\xi)$ are uniformly bounded for $\xi$ real.

ReMARK. This theorem states that $\boldsymbol{P}$ is hyperbolic iff it is properly hyperbolic.

Flaschka and Strang [1] have conjectured that in the general case with constant hyperbolic principal part that the condition that the lower order terms are weaker than the principal part is necessary and sufficient for hyperbolicity. In this paper we will show that this condition is sufficient for hyperbolicity by 
generalizing the method of energy integrals which Peyser devised. Finally we discuss the generalization of the technique to the case of systems of equations and obtain results similar to those of Kopáček and Suchá [5], Svensson [8] and Peyser [7].

2. Acknowledgements. The author wishes to express his appreciation to Martin D. Kruskal, Donald C. Spencer and Arthur S. Wightman for many helpful conversations during the course of this research and to the National Science Foundation for their financial support in the form of a Graduate Fellowship.

3. Statement of the problem. We wish to consider the case of a partial differential operator with constant principal part, i.e.,

$$
P=P_{m}(D)+\sum_{|\alpha|<m} a_{\alpha}(t, x) D^{a}, \text { where } a_{\alpha}(t, x) \in B\left(\mathbf{R}^{n+1}\right),
$$

that is, the space of infinitely differentiable functions which are bounded along with all of their derivatives. We make the further assumption that the principal part $P_{m}(D)$ is hyperbolic in the time direction and that at each point the lower order part is weaker than $P_{m}(D)$.

An example of the type of problem which we wish to solve is given $f \in E^{\prime}\left(\mathbf{R}^{n+1}\right)$ find a $u \in D^{\prime}\left(\mathbf{R}^{n+1}\right)$ such that $P u=f$ and supp $u \subset K+L$ where $K$ is a compact set and $L$ is a proper cone. The obvious way to go about solving this problem is to write the equation as

$$
P_{m}(D) u=-\sum_{|\alpha|<m} a_{\alpha}(t, x) D^{\alpha} u+f
$$

and then perform the implied iteration. In order to show that this iteration converges we will have to use the energy integrals which are the subject of $\S 4$.

We will also be able to determine the regularity properties of $u$ from those of $f$. In fact, if $f \in E^{\prime}\left(\mathbf{R}^{n+1}\right) \cap H^{(s)}\left(\mathbf{R}^{n+1}\right)$ where $H^{(s)}\left(\mathbf{R}^{n+1}\right)$ is the Sobolev space as defined in Hörmander $[4$, p. 45] and $s$ is an integer, then we have $u \in\left(H^{(s)}\right)^{\text {loc }}\left(\mathbf{R}^{n+1}\right)$.

To obtain these results, we will consider the Cauchy problem with homogeneous initial data in a slab $V_{T}=\{(t, x): 0 \leqslant t \leqslant T\}$. Define $D_{+} \subset C^{\infty}\left(R^{n+1}\right)$ to be the set of functions $u$ such that supp $u \cap\{(t, x): t \leqslant 0\}=\varnothing$ and supp $u \subset K+L$ where $K$ is compact and $L$ is a proper cone. Similarly define $D_{-} \subset C^{\infty}\left(\mathrm{R}^{n+1}\right)$ to be the set of functions $u$ such that supp $u \cap$ $\{(t, x): t \geqslant T\}=\varnothing$ and supp $u \subset K-L$ again where $K$ is compact and $L$ is a proper cone. Figures 1 and 2 illustrate the support properties of functions in $D_{+}$and $D_{-}$respectively. 


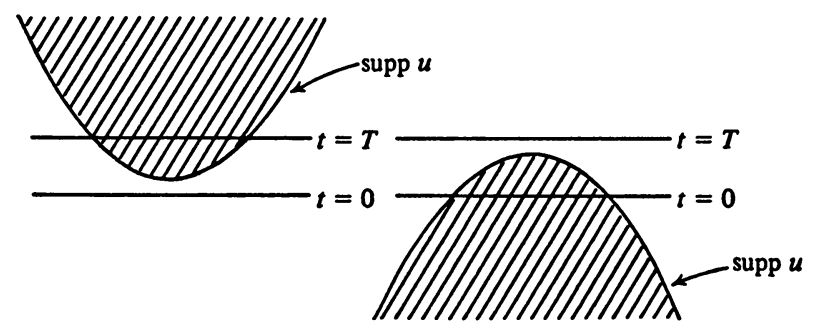

Figure 1. $u \in D_{+} \quad$ FigURE 2. $u \in D_{-}$

DEFINITION 3.1. $H_{+}^{(s)}\left(V_{T}\right), s$ a nonnegative integer, is the completion of $D_{+}$in the norm

$$
\|v\|_{s}=\left(\int_{V_{T}} \sum_{|\alpha|<s}\left|D^{\alpha} v\right|^{2}\right)^{1 / 2}
$$

Now for $u \in D_{+}, s>0$, define

$$
\|u\|_{-s}=\sup _{v \in D_{-}}\left|\int_{V_{T}} u \bar{v}\right| /\|v\|_{s} \leqslant\|u\| .
$$

Definition 3.2. $H_{+}^{(s)}\left(V_{T}\right), s$ a negative integer is the completion of $D_{+}$ in the $\|\cdot\|_{s}$ norm.

DEFinition 3.3. If $f \in H_{+}^{(s)}\left(V_{T}\right), s$ an integer, then we will say $u \in$ $H_{+}^{(s)}\left(V_{T}\right)$ is a strong solution of $P u=f$ with homogeneous initial data, if there exists a sequence $u_{n} \in D_{+}$such that $P u_{n} \rightarrow f$ and $u_{n} \rightarrow u$ both in $H_{+}^{(s)}\left(V_{T}\right)$.

Now given our assumptions on $P$ we will be able to show that for all $f \in i_{+}^{(s)}\left(V_{T}\right)$ there exists a unique strong solution $u \in H_{+}^{(s)}\left(V_{T}\right)$, and that for this solution supp $u \subset \operatorname{supp} f+\Gamma^{*}\left(P_{m},(1,0)\right)$. Using this result one can easily obtain the result for $f \in E^{\prime}\left(\mathbf{R}^{n+1}\right)$ and $u \in \mathcal{D}^{\prime}\left(\mathbf{R}^{n+1}\right)$. These existence theorems will be the subject of $\S 5$. In $\S 6$, we will make an easy generalization of these results to systems of differential operators.

4. Construction of energy integrals. Suppose that $P$ is an operator which satisfies the assumptions of the previous section, in particular $P_{m}(D)$ is assumed hyperbolic. Since the set of differential operators of degree $i$ which are weaker than $P_{m}$ is a linear space, we can choose a basis $p_{i}^{1}, \cdots, p_{i}^{n_{i}}$ for this set. Then our assumptions about $\boldsymbol{P}$ imply that

$$
P u=P_{m}(D) u+\sum_{i=0}^{m-1} \sum_{j=1}^{n_{i}} c_{i}^{j}(t, x) p_{i}^{j}(D) u
$$

where the $c_{i}^{j}(t, x) \in B(t, x)$. Furthermore it is obvious that any operator of form (4.1) satisfies our hypotheses. 
We will now construct energy integrals for operators of form (4.1) following the method of Peyser [6]. Consider the quantity

$$
-\operatorname{Im}\left(P_{m}^{(i-1)}(D) u \overline{\left.P_{m}^{(i)}(D) u\right)} .\right.
$$

One has by methods identical to those of Peyser that

$$
-\operatorname{Im}\left(P_{m}^{(i-1)}(D) u \overline{\left.P_{m}^{(i)}(D) u\right)}=\sum_{j=0}^{m} \partial A_{j}^{m-i}(u) / \partial x_{j}\right.
$$

where the $A_{j}^{m-i}$ are Hermitian forms in the derivatives of order $m-i$ of $u$, and for $u \in D_{+}$or $D_{-}$,

$$
\int_{S_{T^{\prime}}} A_{0}^{m-i}(u) d x \geqslant 0
$$

where $S_{T^{\prime}}$ is the hyperplane $\left\{(t, x): t=T^{\prime}\right\}$.

Lemma 4.1. If $P$ satisfies our hypothesis and $u \in D_{+}$or $D_{-}$then there is a constant $c$ such that

$$
\int_{S_{T^{\prime}}}\left|\sum_{j=1}^{n_{i}} c_{i}^{j}(t, x) p_{i}^{j}(D) u\right|^{2} \leqslant c \int_{S_{T}, A_{0}^{i}(u) .}
$$

Proof.

$$
\begin{aligned}
\int_{S_{T^{\prime}}}\left|\sum_{j=1}^{n_{i}} c_{i}^{j}(t, x) p_{i}^{j}(D) u\right|^{2} & \leqslant \int_{S_{T^{\prime}}}\left(\sum_{j=1}^{n_{i}}\left|c_{i}^{j}(t, x)\right|^{2}\right)\left(\sum_{j}\left|p_{i}^{j}(D) u\right|^{2}\right) \\
& \leqslant c \int_{S_{T^{\prime}}} \sum_{j}\left|p_{i}^{j}(D) u\right|^{2} .
\end{aligned}
$$

The result now follows from Lemma 3 of Peyser [6].

We are now ready to state the main energy inequality.

THEOREM 4.2. If $P$ is of form 4.1, then for each nonnegative integer $s$ there exists a constant $c$ which depends on $P_{m}, T$ and the bounds of the $c_{i}^{j}$ and their derivatives up to order $s$ such that for $T^{\prime}$ in the interval $[0, T]$ and $u \in D_{+}$we have

$$
\sum_{|\gamma|<s} \sum_{i=1}^{m} \int_{S_{T^{\prime}}} A_{0}^{m-i}\left(D^{\gamma} u\right) \leqslant c \sum_{|\gamma|<s} \iint_{T^{\prime}}\left|D^{\gamma} P u\right|^{2} .
$$

Proof. By integrating (4.2) over $V_{T^{\prime}}$ and using the fact that $|\operatorname{Im}(a b)|$ $\leqslant 1 / 2\left(|a|^{2}+|b|^{2}\right)$ we obtain 


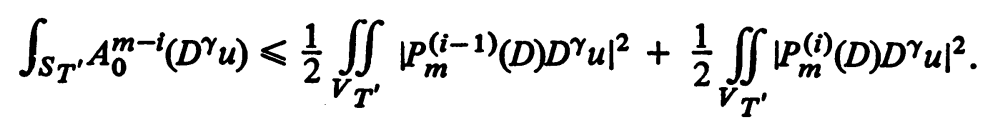

Summing over $i$ and using Lemma 4.1 we obtain

$$
\sum_{i=1}^{m} \int_{S_{T^{\prime}}} A_{0}^{m-i}\left(D^{\gamma} u\right) \leqslant \frac{1}{2} \iint_{V_{T^{\prime}}}\left|P_{m}(D) D^{\gamma} u\right|^{2}+c \sum_{i=1}^{m} \iint_{T_{T^{\prime}}} A_{0}^{m-i}\left(D^{\gamma} u\right) .
$$

Summing over $\gamma$ for $|\gamma| \leqslant s$ gives

$$
\sum_{|\gamma|<s} \sum_{i=1}^{m} \int_{S_{T^{\prime}}} A_{0}^{m-i}\left(D^{\gamma} u\right)
$$

Now

$$
\leqslant \frac{1}{2} \sum_{|\gamma|<s} \iint_{V_{T^{\prime}}}\left|P_{m}(D) D^{\gamma} u\right|^{2}+c \sum_{|\gamma|<s} \sum_{i=1}^{m} \iint_{T_{T^{\prime}}} A_{0}^{m-i}\left(D^{\gamma} u\right) .
$$

$$
P_{m} D^{\gamma} u=D^{\gamma} P_{m} u=D^{\gamma} P u-D^{\gamma}\left[\sum_{i} \sum_{j} c_{i}^{j}(t, x) p_{i}^{j}(D) u\right]
$$

Applying Leibniz's rule to the quantity in brackets gives

$$
\begin{aligned}
P_{m} D^{\gamma} u & =D^{\gamma} P u-\gamma ! \sum_{\alpha+\beta=\gamma} \sum_{i} \sum_{j}\left(\frac{D^{\alpha} c_{i}^{j}}{\alpha !}\right)\left(\frac{D^{\beta} p_{i}^{j}(D) u}{\beta !}\right) \\
& =D^{\gamma} P u-\gamma ! \sum_{\alpha+\beta=\gamma} \sum_{i} \sum_{j}\left(\frac{D^{\alpha} c_{i}^{j}}{\alpha !}\right)\left(\frac{p_{i}^{j}(D) D^{\beta} u}{\beta !}\right) .
\end{aligned}
$$

Thus

$$
\begin{aligned}
\iint_{T_{T^{\prime}}}\left|P_{m} D^{\gamma} u\right|^{2} & \leqslant \iint_{T_{T^{\prime}}}\left|D^{\gamma} P u\right|^{2}+c \sum_{\beta<\gamma} \sum_{i, j} \iint_{V_{T^{\prime}}}\left|p_{i}^{j}(D) D^{\beta} u\right|^{2} \\
& \leqslant \iint_{V_{T^{\prime}}}\left|D^{\gamma} P u\right|^{2}+c \sum_{\beta<\gamma} \sum_{i} \iint_{V_{T^{\prime}}} A_{0}^{m-i}\left(D^{\beta} u\right) \\
& \leqslant \iint_{V_{T^{\prime}}}\left|D^{\gamma} P u\right|^{2}+c \sum_{|\beta|<s} \sum_{i} \iint_{V_{T^{\prime}}} A_{0}^{m-i}\left(D^{\beta} u\right) .
\end{aligned}
$$

Substituting this into (4.5) we obtain

$$
\begin{aligned}
\sum_{|\gamma|<s} & \sum_{i=1}^{m} \int_{S_{T^{\prime}}} A_{0}^{m-i}\left(D^{\gamma} u\right) \\
& \leqslant \frac{1}{2} \sum_{|\gamma|<s} \iint_{V_{T^{\prime}}}\left|D^{\gamma} P u\right|^{2}+c \sum_{|\gamma|<s} \sum_{i=1}^{m} \iint_{V_{T^{\prime}}} A_{0}^{m-i}\left(D^{\gamma} u\right) .
\end{aligned}
$$


Now by a classical result about integral inequalities, this implies,

$$
\sum_{|\gamma|<s} \sum_{i=1}^{m} \int_{s_{T^{\prime}}} A_{0}^{m-i}\left(D^{\gamma} u\right) \leqslant c \sum_{|\gamma|<s} \iint_{T_{T^{\prime}}}\left|D^{\gamma} P u\right|^{2} .
$$

COROLLARY 4.3. If $P$ is of form (4.1), $u \in D_{+}$and $s$ a nonnegative integer, then $\|u\|_{s} \leqslant c\|P u\|_{s}$ where $\|\cdot\|_{s}$ is the norm defined by

$$
\|u\|_{s}^{2}=\sum_{|\gamma|<s} \iint_{V}\left|D^{\gamma} u\right|^{2} .
$$

In order to obtain results about the existence of solutions of the equation $P u=f$ for $f \in H_{+}^{(s)}\left(V_{T}\right)$ when $s$ is negative, we will need energy inequalities for the adjoint of $P, P^{*}$ :

$$
\begin{aligned}
P^{*} u & =\overline{P_{m}(-D)} u+\sum_{i} \sum_{j} \overline{p_{i}^{j}(-D) c_{i}^{j}(t, x)} u \\
& =P_{m}(D) u+\sum_{\alpha} \sum_{i} \sum_{j}\left[(-D)^{\alpha} \bar{c}_{i}^{j}\right] \overline{\left(p_{i}^{j}\right)^{(\alpha)}(-D)} u
\end{aligned}
$$

Now $\left(p_{i}^{j}\right)^{(\alpha)}$ is weaker than $P_{m}$ if $p_{i}^{j}$ is and so the verification that $P^{*}$ satisfies our hypothesis is reduced to the following: Claim if $p$ is weaker than $P_{m}(D)$ then $\overline{p(-D)}$ is weaker than $P_{m}(D)$. For if $p(D)=\Sigma a_{\alpha} D^{\alpha}$, then $p(-D)=\Sigma a_{\alpha}(-D)^{\alpha}$ and $\overline{p(-D)}=\Sigma \bar{a}_{\alpha} D^{\alpha}$. Thus if $f(\xi)$ is the polynomial corresponding to $\overline{p(-D)}$, then $f(\xi)=\overline{p(\xi)}$ for $\xi$ real and thus $|f(\xi)|=|p(\xi)|$. Now by Theorem 3.3.2(a) of Hörmander [4] this implies that $\overline{p(-D)}$ is weaker than $P_{m}$ if $p(D)$ is. Thus $P^{*}$ satisfies our hypothesis. One can now prove the analogs of Theorem 4.2 and Corollary 4.3 with $u \in D_{+}$replaced by $u \in D_{-}$ and $\boldsymbol{P}$ by $\boldsymbol{P}^{*}$.

5. Existence theory. We are now ready to prove existence theorems for the Cauchy problem for $P u=f$ with homogeneous initial data.

THEOREM 5.1. If $P$ is of form (4.1) and $f$ is an arbitrary element of $H_{+}^{(s)}\left(V_{T}\right), s$ an integer, then there exists a unique strong solution $u \in H_{+}^{(s)}\left(V_{T}\right)$ of the problem $P u=f$ with homogeneous initial data. We have for this $u$ that supp $u \subset \operatorname{supp} f+\Gamma^{*}\left(P_{m},(1,0)\right)$.

The proof of this result will proceed in several steps. Step 1 is the proof of the result for nonnegative $s$. In the course of this proof we will show that for every $f \in D_{+}$there is a unique $u \in D_{+}$such that $P u=f$. A similar result also holds for $u$ and $f \in D_{-}$and $P^{*} u=f$. Step 2 uses the results of Step 1 to obtain estimates in $H_{+}^{(s)}$ for $s$ negative which can then be used to prove the result for such $s$. 
STEP 1. Assume that $s$ is a nonnegative integer. Uniqueness is easy. If $u$ and $v$ are two strong solutions corresponding to $f$, then there exist two sequences $u_{n}$ and $v_{n}$ such that $P u_{n} \rightarrow f, u_{n} \rightarrow u, P v_{n} \rightarrow f$, and $v_{n} \rightarrow v$ all in $H_{+}^{(s)}\left(V_{T}\right)$. This implies that $P\left(u_{n}-v_{n}\right) \rightarrow 0$ in $H_{+}^{(s)}\left(V_{T}\right)$ and thus by Corollary $4.2 u_{n}-v_{n} \rightarrow 0$. Therefore $u=v$.

For the existence of a strong solution $u$ of the problem it suffices to find a sequence $u_{n} \in D_{+}$such that $P u_{n} \rightarrow f$. Indeed, if $u_{n}$ is such a sequence, Corollary 4.2 shows that $u_{n}$ is Cauchy and hence must converge in $i f_{+}^{(s)}\left(V_{T}\right)$. Suppose now that one has the following

Lemma 5.2. If $P$ is of form (4.1) and $g \in D_{+}$then there exists a unique $u \in D_{+}$such that $P u=g$. Furthermore we have for this $u$ that $\operatorname{supp} u$ $C$ supp $g+\Gamma^{*}\left(P_{m},(1,0)\right)$.

Now by choosing $f_{n} \in D_{+}$such that $\left\|f_{n}-f\right\|_{s}<\epsilon=1 / n$ and supp $f_{n}$ $C \operatorname{supp} f+B_{\epsilon}$ where $B_{\epsilon}$ is the ball of radius $\epsilon$ about 0 , we have for the $u_{n}$ given by the lemma that $P u_{n}=f_{n}$ and that supp $u_{n} \subset \operatorname{supp} f_{n}+\Gamma^{*} \subset$ supp $f+\Gamma^{*}+B_{\epsilon}$ and thus the limit of the $u_{n}, u$ has supp $u \subset \operatorname{supp} f+\Gamma^{*}$, and is a strong solution of $P u=f$.

Proof of lemMA. We will perform the iteration

$$
\begin{aligned}
u_{0} & =0 \\
P_{m} u_{l+1} & =g-\sum_{i} \sum_{j} c_{i}^{j}(t, x) p_{i}^{j}(D) u_{l} .
\end{aligned}
$$

That is,

$$
u_{l+1}=E *\left(g-\sum_{i} \sum_{j} c_{i}^{j}(t, x) p_{i}^{j}(D) u_{l}\right)
$$

where $E$ is the fundamental solution of $P_{m}(D)$ with support in the cone $\Gamma^{*}\left(P_{m},(1,0)\right)$.

Claim that all the $u_{l}$ are in $D_{+}$and that in fact supp $u_{l} \subset \operatorname{supp} g+$ $\Gamma^{*}\left(P_{m},(1,0)\right)$. Clearly this is true for $u_{0}$. If it is true for $u_{l}$ then $g-$ $\Sigma_{i} \Sigma_{j} c_{i}^{j} p_{i}^{j}(D) u_{l} \in D_{+}$and thus by elementary facts about convolutions $u_{l+1} \in$ $D_{+}$. Furthermore

$$
\begin{aligned}
\operatorname{supp} u_{l+1} & \subset \operatorname{supp} E+\left(\operatorname{supp} g \cup \operatorname{supp} u_{l}\right) \\
& =(\operatorname{supp} E+\operatorname{supp} g) \cup\left(\operatorname{supp} E+\operatorname{supp} u_{l}\right) \\
& \subset\left(\Gamma^{*}\left(P_{m}(1,0)\right)+\operatorname{supp} g\right) \cup\left(\Gamma^{*}\left(P_{m},(1,0)\right)+\Gamma^{*}\left(P_{m},(1,0)\right)+\operatorname{supp} g\right) \\
& =\left(\Gamma^{*}+\operatorname{supp} g\right) \cup\left(\Gamma^{*}+\operatorname{supp} g\right)=\Gamma^{*}+\operatorname{supp} g
\end{aligned}
$$


where we have used the fact that $\Gamma^{*}$ is a convex cone. Thus if these $u_{l}$ have a limit in $D_{+}$it is supported on $\Gamma^{*}+$ supp $g$.

We will now use the energy inequalities of the previous section. One has

$$
\begin{aligned}
P_{m}(D)\left(u_{1}-u_{0}\right) & =g \\
P_{m}(D)\left(u_{l+1}-u_{l}\right) & =-\sum c_{i}^{j} p_{i}^{j}(D)\left(u_{l}-u_{l-1}\right) .
\end{aligned}
$$

Now for $T^{\prime}$ fixed, $t$ in the interval $\left[0, T^{\prime}\right]$ and

$$
G_{l}(t) \equiv \sum_{|\gamma|<s} \sum_{i=1}^{m} \int_{S_{t}} A_{0}^{m-i}\left(D^{\gamma}\left(u_{l+1}-u_{l}\right)\right)
$$

we have that

$$
G_{0}(t) \leqslant c \sum_{|\gamma|<s} \iint_{V_{t}}\left|D^{\gamma} g\right|^{2} \leqslant c_{1}
$$

and

$$
\begin{aligned}
G_{l}(t) & \leqslant c \int_{0}^{t} d t^{\prime} \sum_{|\gamma|<s} \sum_{i=1}^{m} \int_{S_{t^{\prime}}}\left|D^{\gamma} \sum c_{i}^{j} p_{i}^{j}(D)\left(u_{l}-u_{l-1}\right)\right|^{2} \\
& \leqslant c_{2} \int_{0}^{t} d t^{\prime} G_{l-1}\left(t^{\prime}\right) .
\end{aligned}
$$

One obtains easily that $G_{l}(t) \leqslant c_{1}\left(c_{2} t\right)^{l} / l !$ and therefore $G_{l}(t) \rightarrow 0$ uniformly for $0 \leqslant t \leqslant T^{\prime}$. Furthermore

$$
\begin{aligned}
\sum_{|\gamma|<s} \int_{V_{T^{\prime}}}\left|D^{\gamma}\left(u_{l}-u_{k}\right)\right|^{2} & \leqslant c \int_{0}^{T^{\prime}} d t^{\prime} \sum_{i=k}^{l-1} G_{i}\left(t^{\prime}\right) \\
& \leqslant c T^{\prime} \sum_{i=k}^{l-1} \frac{\left(c_{2} T^{\prime}\right)^{i}}{i !}
\end{aligned}
$$

which implies that $u_{l}$ is Cauchy in the $H_{+}^{(s)}\left(V_{T^{\prime}}\right)$ norm. Letting $s$ and $T^{\prime}$ go to infinity one obtains that $u_{l}$ converges in $C^{\infty}\left(R^{n+1}\right)$ to an element of $D_{+}$. This completes the proof of the lemma.

STEP 2. Suppose now that $s$ is a negative integer and that $f \in f_{+}^{(s)}\left(V_{T}\right)$. The proof will go through much as before if we can establish the extension of Corollary 4.3 to the case of negative $s$.

For $f \in D_{-}$denote by $E f$ the unique element in $D_{-}$such that $P^{*} E f=f$. Then we have for $u \in D_{+}$ 


$$
\begin{aligned}
\|P u\|_{s} & =\sup _{v \in D_{-}}|(v, P u)| /\|v\|_{-s}=\sup _{v \in D_{-}}\left|\left(P^{*} v, u\right)\right| /\|v\|_{-s} \\
& =\sup _{f \in D_{-}}\left|\left(P^{*} E f, u\right)\right| /\|E f\|_{-s}=\sup _{f \in D_{-}}|(f, u)| /\|E f\|_{-s} .
\end{aligned}
$$

But by Corollary $4.2,\|E f\|_{-s} \leqslant c\|f\|_{-s}$ and thus

$$
\|P u\|_{s} \geqslant c \sup _{f \in D_{-}}|(f, u)| /\|f\|_{-s}=c\|u\|_{s} .
$$

This completes the proof of Theorem 5.1.

Let us now consider the problem $P u=f$ where $f \in E^{\prime}\left(R^{n+1}\right)$ and where $u \in D^{\prime}\left(\mathrm{R}^{n+1}\right)$ with supp $u \subset K+L, K$ compact and $L$ a proper cone. The assumption that $f \in E^{\prime}$ implies that $f \in H^{(s)}\left(\mathbf{R}^{n+1}\right)$ for some $s$. We can by choosing the origin of time appropriately assure that $\operatorname{supp} f \cap\{(t, x): t \leqslant 0\}=$ $\varnothing$, and we can choose $T$ large enough so that $\operatorname{supp} f \cap\{(t, x): t \geqslant T\}=\varnothing$. Then we have by methods similar to those of Hörmander $[4, \S 2.5]$ that $f \in$ $H_{+}^{(s)}\left(V_{T}\right)$ and

$$
\|f\|_{H+\left(V_{T}\right)}=\|f\|_{H(s)}\left(\mathrm{R}^{n+1}\right)^{\cdot}
$$

Thus we have a unique strong solution $u \in H_{+}^{(s)}\left(V_{T}\right)$. Furthermore, we also have a strong solution in $H_{+}^{(s)}\left(V_{T^{\prime}}\right)$ for $T^{\prime}>T$ which can be restricted to $\psi_{+}^{(s)}\left(V_{T}\right)$ to yield the already given solution. Proceeding in this manner one can construct a $u \in H^{(s) \text { loc }}\left(\mathbf{R}^{n+1}\right)$ with $u=0$ for $t<0$ such that $P u=f$ and such that supp $u \subset \operatorname{supp} f+\Gamma^{*}$. Furthermore, it is easy to show that among the elements of $D^{\prime}\left(\mathrm{R}^{n+1}\right)$ such that $P u=f$ and supp $u \subset K+L, K$ compact and $L$ a proper cone, the solution is unique. Summarizing we have

THEOREM 5.3. If $P$ satisfies our hypothesis and $f \in E^{\prime}\left(R^{n+1}\right)$ then the equation $P u=f$ has a solution $u \in D^{\prime}\left(R^{n+1}\right)$ with supp $u \subset \operatorname{supp} f+$ $\Gamma^{*}\left(P_{m},(1,0)\right)$ and this solution is unique in the class of $u \in D^{\prime}\left(R^{n+1}\right)$ with supp $u \subset K+L$ where $K$ is compact and $L$ is a proper cone. One has also that $f \in H^{(s)}$ implies $u \in H^{(s) l o c}$, where $s$ is an arbitrary integer.

6. Systems. We will now suppose that we have a square system of differential equations $P u=\Sigma P_{i j}(D) u_{j}=f_{i}$. Certain special operators of this type will admit a treatment similar to the one we have already given for scalar operators. The most obvious such operators are of the form $P u=P_{m}(D) I+$ lower order terms where $P_{m}(D)$ is a scalar operator with constant coefficients which is homogeneous of degree $m$ and hyperbolic in the time direction. If one makes the further assumption that for each matrix position the lower order terms are 
weaker than $P_{m}$, then one can push through the analogs of all the theorems of $\S \S 4$ and 5. For instance,

Theorem 6.1. If $P u=P_{m}(D) u+Q u$ where $P_{m}$ is a scalar operator homogeneous of degree $m$ with constant coefficients and hyperbolic in the time direction and $Q$ is a matrix of differential operators with coefficients in $B$ such that at each point $Q_{i j}$ is weaker than $P_{m}$, then for each $f \in H_{+}^{(s)}\left(V_{T}\right)$, $s$ an integer, there exists a unique $u \in H_{+}^{(s)}\left(V_{T}\right)$ such that $u$ is a strong solution of $P u=f$ with homogeneous initial data. Furthermore supp $u \subset \operatorname{supp} f+$ $\Gamma^{*}\left(P_{m},(1,0)\right)$.

We also have for such $P$ that if $f \in E^{\prime}$ then there exists a unique $u \in D^{\prime}$ such that $P u=f$ and $\operatorname{supp} u \subset K+L, K$ compact and $L$ a proper cone. For this $u$ we have supp $u \subset \operatorname{supp} f+\Gamma^{*}\left(P_{m},(1,0)\right)$.

The proof of this theorem and of all the other theorems from $\S \S 4$ and 5 go through virtually without change.

One can obtain results for other systems of equations when it is possible to throw them into the above form. For instance if there exists an operator $R$ such that $R P$ is in the above form, then one has that for any $f \in E^{\prime}$ there exists at most one $u \in D^{\prime}$ with supp $u \subset K+L, K$ compact and $L$ a proper cone such that $P u=f$. Similarly if $P R$ is in the above form one obtains an existence theorem.

Finally let us consider the problem when $P=A+B$ where $A$ is a matrix of differential operators with constant coefficients. One makes the further assumption that there exists another operator $C$ again with constant coefficients such that $C A=p(D) I$ where $p$ is a hyperbolic polynomial and such that $C B$ is weaker than the principal part of $p$. Clearly one has uniqueness for such an equation. One has existence also. In fact, if $C P u=C f$ where $f \in E^{\prime}$ and $u \in D^{\prime}$ with supp $u \subset K+L, K$ compact and $L$ a proper cone then $P u=f$. For $C(P u-f)=0$ with supp $(P u-f) \subset K+L$, therefore since our assumptions on $C$ imply that it is a hyperbolic operator we have $P u=f$. To see why $C$ is hyperbolic one needs the result of Gårding and Svensson [8], that a matrix of operators with constant coefficients is hyperbolic iff its determinant is hyperbolic. However, that $C$ has hyperbolic determinant follows easily from the fact that $C P=p I$ where $p$ is a hyperbolic polynomial.

\section{BIBLIOGRAPHY}

1. H. Flaschka and G. Strang, The correctness of the Cauchy problem, Advances in Math. 6 (1971), 347-379. MR 43 \#5147.

2. L. Gårding, Linear hyperbolic partial differential equations with constant coefficients, Acta. Math. 85 (1951), 1-62. MR 12, 831. 
3. L. Gårding, Solution directe du problème de Cauchy pour les équations hypérboliques, La théorie des équations aux dérivées partielles (Nancy, 1956), Colloq. Internat. du Centre National de la Recherche Scientifique, 71, Paris, 1956, pp. 71-90. MR 22 \#6937.

4. L. Hörmander, Linear partial differential operators, 3rd rev. ed., Die Grundlehren der math. Wissenschaften, Band 116, Springer-Verlag, New York, 1969. MR 40 \#1687.

5. J. Kopáček and M. Suchá, The Cauchy problem for weakly hyperbolic systems of linear differential equations with constant coefficients, Casopis Pést. Mat. 91 (1966), 431 452. (Russian) MR 34 \#7969.

6. G. Peyser, Energy inequalities for hyperbolic equations in several variables with multiple characteristics and constant coefficients, Trans. Amer. Math. Soc. 108 (1963), 478-490. MR 28 \#352.

7. - Hyperbolic systems with multiple characteristics, J. Differential Equations 9 (1971), 509-520. MR 43 \#6593.

8. S. L. Svensson, Necessary and sufficient conditions for the hyperbolicity of polynomials with hyperbolic principal part, Ark. Mat. 8 (1969), 145-162. MR 42 \#6421.

DEPARTMENT OF MATHEMATICS, PRINCETON UNIVERSITY, PRINCETON, NEW JERSEY 08540 